\title{
HILOS CONDUCTORES DEL APRENDIZAJE AUTÓNOMO Y LOS PROCESOS DE RAZONAMIENTO
}

\author{
Luis Facundo Maldonado Granados ${ }^{*}$ \\ Nerey Ortega del Castillo* \\ Luis Bayardo Sanabria Rodríguez * \\ David Macías Mora *
}

\begin{abstract}
TECNICE is a research group housed in the Universidad Pedagógica Nacional, focused on use of computerprograms for the understanding an development of autonomous learning. Along its research evolution has been dealing with the learning domains of spatial reasoning, industrial design, andgeography. Meaning ful learning and learning strategies are psychological core topics in the studies conducted. Autonomy is explained in the framework of metacognition from the point of view on metamemory judgments, problem solving strategies and knowledge representation structures. Its epistemological and methodological approach includes qualitative as well as quantitative logic, simulation processes and ontological representation. From de stand point of view of technological developments the results include discovery games, software agants to the production of structured hypertexts, simulators of problem solving process. question generator and $Q$ agents.
\end{abstract}

\section{RESUMEN}

TECNICE, con sede en la Universidad Pedagógica Nacional, se constituye como grupo de investigación centrado en el uso de programas de computador para comprender y desarrollar la autonomía en el aprendizaje. Su evolución conceptual le ha llevado a integrar desarrollos que tienen, como dominio de conocimiento el razonamiento espacial en el contexto del diseño y del conocimiento geográfico que incorporan dimensiones del aprendizaje significativo y estrategias de colaboración. La autonomía se interpreta desde la óptica de la metacognición y más específicamente desde los: juicios de metamemoria, las estrategias de solución de problemas y las estructuras de representación. Su posición epistemológica y metodologica incorpora lógicas cualitativas y cuantitativas, procesos de simulación y representación ontológica. Sus desarrollos tecnológicos se centran en juegos de descubrimiento, agentes de software para generación de hipertextos estructurados, simuladores de procesos de solución de problemas, generadores de preguntas y agentes Q.

Palabras clave: Metacognición, autonomía, aprendizaje, agentes de software, razonamiento espacial, aprendizaje por descubrimiento, ontologías, diseño de software.

\footnotetext{
* Profesores investigadores Grupo Tecnice. Universidad Pedagógica Nacional.
} 
El interés del grupo TECNICE es el de modelar el mundo do la educación mediante un juego dinámico entre los enfoques cualitativo y cuantitativos. El estudio de lo metacognitivo realza la autonomía como dimensión básica del trabajo creativo. En la práctica se integran intereses lógicos, estéticos, éticos y lúdicos en la generación de soluciones informáticas para la educación.

\section{Introducción: línea de investigación}

La comprensión de los procesos que siguen los estudiantes para construir autonomía en el aprendizaje, ha sido, desde sus inicios, el interés dominante del grupo TECNICE Tecnologías de la Información y la Comunicación Aplicadas a la Educación- Nuestros proyectos consideran de manera explícita categorías de aprendizaje, dominio de conocimiento y un tipo de ambiente de programa de computador.

Metacogníción y redes conceptuales son categorías de aprendizaje que dominan el interés de nuestros proyectos. La metacognición la hemos estudiado desde la perspectiva de los juicios de metamemoria y las estrategias de solución de problemas. Las redes conceptuales han sido de nuestro interés por su relación con el aprendizaje significativo en los siguientes tópicos: construcción de sistemas conceptuales, riqueza de conexiones en redes de conceptos, inferencia y profundidad en el razonamiento.

El razonamiento espacial ha sido dominio de conocimiento desde la primera etapa, con proyecciones al diseño, el arte, la historia de los objetos y la geografía. El aprendizaje sobre derechos fundamentales constituye la segunda área temática sobre la cual nos venimos ocupando recientemente.

Los escenarios de experimentación son las ambientes de aprendizaje basados en computador con aplicaciones a las aulas de clase de la educación formal. Los programas desarrollados por el grupo combinan ambientes de descubrimiento, hipertextos, simuladores de procesas, ambientes para que los estudiantes representen conocimiento, generadores de preguntas y agentes de software para el juego de roles.

\section{Trabajos que originaron la línea}

Maldonado, Monroy, Carrillo y Terrero (1 978), desarrollan un trabajo sobre ambientes de aprendizaje, enseñanza personalizada y uso de documentos escritos. Prueban el efecto de los materiales textuales en el aprendizaje, valoran métodos como la enseñanza personalizada basada en estudio personal y entrevistas y muestran que los sistemas de aprendizaje autónomo pueden ser tan buenos o mejores que los controlados directamente parlas profesores mediante interacción sincrónica con los grupos de estudiantes.

Maldonado y Ochoa (1984), elaboran un estado del arte sobre el tema de las tecnologías en la educación y, con base en éste, presentan una primera propuesta de programa de magíster. El enfoque se orienta a relacionar disciplinas como: la semiótica, la comunicación, la teoría matemática de la información con los desarrollos de la inteligencia artificial aplicadas a la comprensión de procesos de pensamiento. 
Maldonado (1990), presenta a la UNESCO el proyecto de 'tecnologías de la información y la comunicación para la educación TECNICE" con una prospectiva de diez años. El proyecto se desarrolla en colaboración con las Universidades

Complutense y Politécnica de Madrid y se organiza el Centro de Informática de la Universidad Pedagógica, CIDUP, que se constituye en ambiente propicio para la investigación de procesos de aprendizaje y que da origen al grupo de investigación que asume el nombre de grupo

TECNICE.

En la consolidación del grupo se vivieron dos fases: en la primera confluyen las líneas texto, vídeo, periodismo en ciencias, informática para personas con discapacidades y aprendizaje autónomo; en la segunda se fortalece esta última línea.

En 1996 se presenta a Colciencias el primer proyecto de investigación para estudiar el efecto del feedback sobre el desarrollo de las capacidades de autoevaluación del aprendizaje en problemas de razonamiento espacial y diseño gráfico. Los escenarios de experimentación son juegos de descubrimiento basados en computador.

Con este proyecto se identifican la cognición, la metacognición, el razonamiento espacial y la resolución de problemas, como categorías esenciales del aprendizaje autónomo y sobre las cuales girará el desarrollo de proyectos posteriores que consolidan las bases epistemológicas y metodológicas de los procesos de investigación.

\section{Fundamentos epistemológicos}

La fundamentación de la línea de investigación se basa en cinco posiciones epistemológicas:

1. Representar es comprender.

2. La estadística apoya la generalización mediante la normalización.

3. Las lógicas cualitativa y cuantitativa son dimensiones complementarias en una misma representación del mundo.

4. El mundo existe a través de ontologías.

5. La investigación experimental es fuente de metáforas para orientar la acción en los entornas naturales.

\section{Representar es comprender}

La ciencia no es otra cosa que sistemas de representaciones del mundo, construidas ya sea mediante el acercamiento analítico propio de las ciencias de la naturaleza y de la sociedad o la síntesis que domina en las ciencias de lo artificial. Las representaciones científicas que históricamente se han basado en códigos verbales, cuentan ahora con ¡adinámica del computador que les permite utilizar la simulación de procesos para mejorar su comprensión. La virtualidad inherente a formas de arte como el teatro o el cine incrementa su fuerza con la interactividad resultante de la programación. El computador, además de permitir replicar procesos, permite entender sus estructuras para hacer prospectiva. En particular, frente al aprendizaje y los sistemas educativos, el modelamiento de sistemas y procesos permite incrementar nuestro conocimiento sobre la naturaleza de la inteligencia humana y la dinámica de la educación en la sociedad. 


\section{La estadística apoya la generalización mediante la normalización}

El estudio intensivo de los procesos en los individuos nos permite establecer una base muy sólida para entender los mecanismos de aprendizaje. Para algunos, esta aproximación da suficiente validez a las investigaciones. Sin embargo, queda pendiente la pregunta sobre la representatividad de sus conclusiones:¿basta entender un individuo para entender a toda la especie? En el grupo, aceptamos que la comparación de grupos fortalece la generalización de las conclusiones. Si los rasgos estudiados en los individuos se miran desde la perspectiva de su distribución normal, nuestra comprensión de los fenómenos se ve fortalecida. Los modelos de análisis estadístico son mecanismos para potenciar los procesos de inducción en la construcción de generalizaciones. Nuestro enfoque parte de un estudio intensivo de los procesos individuales y procede a ampliar la extensión de los conceptos construidos evaluando su distribución normal mediante modelos estadísticos.

\section{Las lógicas cualitativa y cuantitativa son dimensiones complementarias en una misma representación del mundo}

La tercera posición establece la relación entre las lógicas cualitativa y cuantitativa en el proceso de investigación. Las dos formas de análisis experimental exploran hechos observados en ópticas diferentes que se complementan para buscar la respuesta a un mismo problema. En tanto la lógica cualitativa se centra en la diferenciación, la cuantitativa se centra en la métrica. Regularmente, la primera antecede a la segunda; por ejemplo, las relaciones de giro a derecha o izquierda anteceden a la inclusión del grado como indicador cuantitativo. En los procesos las relaciones de secuencia son base para la identificación de operandos, operadores o resultados y, a su vez, éstos sustentan la construcción de una métrica apropiada para cuantificar dimensiones de los procesos. El razonamiento espacial cualitativo genera modelos consistentes que complementados con la lógica cuantitativa permiten la simulación de los procesos de razonamiento seguidos por agentes naturales. Lo común a las dos aproximaciones es la consideración de relaciones de manera consistente. La complementación de los das métodos ha generado un camino para comprender los procesos mentales de los agentes naturales.

\section{El mundo existe a través de ontologías}

La cuarta posición plantea que el mundo o la realidad existe a través de ontologías. Todo ser es potencialmente existente. Existe cuando se devela a otro ser; cuando es un "ser para otro". El "ser para otro" exige en el "otro" la posibilidad de representar. El paso del ser a la existencia es el encuentro con el cognoscente. El investigador por función torna a los seres en existentes y eso lo hace a través de la representación. Una estructura de representación es una ontología. La ontología transforma la sensación en percepción, el estímulo en significado.

Las ontologías como estructuras perceptivas generadoras de significado son perspectivas de representación de los seres. Ejemplos de estas ontologías son las clasificaciones jerárquicas reflejadas en la programación orientada a objetos con las relaciones jerárquicas de herencia, sucesoras de la teoría de conjuntos, con antecedentes en la lógica aristotélica, o la perspectiva de la dinámica de sistemas basada en relaciones de regulación.

En la medida que el investigador desarrolla ontologías puede hacer que la naturaleza se devele y descubra sus secretos y en la medida que esto se hace, mejora su capacidad 
de síntesis, lo cual le permite crear nuevas formas de ordenamiento y dispositivos para potenciar su acción.

\section{La investigación experimental es fuente de metáforas para orientar la acción en los entornos naturales}

La quinta posición considera los entornas experimentales como fuentes de metáforas para construir modelos que permitan optimizar la intervención del entorno natural. Tanto las condiciones de los estudios experimentales como las de las acciones educativas, son resultado de la planeación y el diseño, pero las primeras dejan de lado muchos factores importantes en las segundas. El reto del investigador en educación es hallar el camino más apropiado para proyectar los resultados de la investigación con niveles altos de abstracción en el mejoramiento de la acción cotidiana de mayor complejidad y menor abstracción. Es, en este contexto, donde la generación de modelos, a partir de la investigación experimental para resolver problemas educativos, se torna en estrategia valiosa. Los modelos surgidos de esta forma preservan las relaciones consideradas en los experimentos e incorporan la complejidad de las interacciones de los agentes de los entornas educativos. La lógica de la investigación es la de la verdad probable y la del modelo educativo la del mejoramiento progresivo.

\section{Fundamentos metodológicos}

\section{Análisis de protocolos de reportes verbales}

El interés por entender los procesos que siguen los estudiantes cuando se enfrentan a problemas de descubrimiento, determinó el interés del grupo por la metodología de análisis de protocolos de reportes verbales (Ericson y Simon, 1994). La representación de un problema se concibe como la secuencia de la transiciones de estados generadas cuando el sujeto aplica un operador a un operando. Trabajos desarrollados por Newell \& Simon (1972) dan cuenta de esta posición.

La representación del sujeto expresada en código verbal, cuando habla en voz alta, al tiempo que resuelve un problema, se toma como indicador válido del proceso que sigue. El investigador que usa esta metodología, debe desarrollar varias operaciones: registrar tan fielmente como sea posible, generalmente usando dispositivos de grabación, la expresión del sujeto; identificar la estructura del proceso en términos de estados, operadores y operandas; sintetizar la información identificando subprocesos y estructurando unidades significativas; traducir los segmentos a código de computador y simular los procesos seguidos; finalmente, construir un modelo de solución e interpretar teóricamente la estructura de todo el proceso.

\section{Simuladores de procesos de solución de problemas}

La utilización de análisis de protocolos verbales nos llevó a programar el computador para que construyera una forma de protocolo automatizado. Siguiendo la directriz consistente en identificar operadores, operandos y transiciones de estado, programamos el computador para que tomara los datos de cada paso, preservando la secuencia seguida y el tiempo invertido por los sujetos. Un programa complementario podía replicar la secuencia y apoyar su análisis con operaciones de segmentación, clasificación y optimización. 
La importancia de caracterizar un fenómeno natural mediante una construcción artificial ha llevado a inferir las operaciones mentales que desarrolla quien resuelve un problema (espacio del problema). La simulación se convierte en estrategia para caracterizar los modelos mentales en la medida en que objetiva procesos y los torna reproducibles para su estudio.

\section{Modelo experimental estadístico}

El desarrollo de programas de computador permite desarrollar estudios experimentales que antes eran muy difíciles de llevar a cabo por problemas de control. Si bien es cierto que contrastar un grupo que usa computador con otro que no, genera efectos de halo que afectan la validez interna de las conclusiones, la configuración de diferentes formas de software de acuerdo a las variables independientes de interés, permite trabajar con diseños experimentales propiamente dichos, tales como el diseño experimental con grupo control o el factorial. Las críticas hechas al uso de estos diseños en informática educativa, se han centrado en la duración de los experimentos y en los tamaños de las muestras.

Nuestra estrategia, frente a la primera crítica, la hemos enfrentado de das maneras: por una parte, incrementando el número de unidades experimentales, lo cual hace que podamos trabajar durante períodos de una o das semanas con dos horas diarias de trabajo y, por otra, generando replicación sistemática de los experimentos; es decir, diseñando nuevos experimentos que se orientan a confirmar resultados previamente hallados, introduciendo nuevas preguntas y nuevas variables.

El diseño experimental se complementa con el análisis de protocolos o la simulación de procesos. La lógica del análisis de protocolos es la comprensión de un proceso en unos pocos sujetos, la del diseño experimental estadístico es la de la predicción. Tanto en el análisis factorial de varianza como en la regresión, la relación entre variabilidad en la variable dependiente generada parlas cambios en las variables independientes y la atribuible a otras variables o de error, determina la fuerza de las predicciones.

En resumen, las conclusiones del estudio intensivo, con base en el análisis de protocolos, es valorado en su generalidad o capacidad predictiva con los diseños experimentales estadísticos.

\section{Ontologías}

Una consecuencia del principio epistemológico, según el cual los seres existen a través de ontologías, es su aplicación a la adquisición y representación de conocimiento en programas de computador. Los trabajos de Minsky (1974) y Sowa (1986), sobre redes semánticas y redes conceptuales, generaron una tradición importante en ingeniería del conocimiento. Nuestro grupo ha venido aplicando este enfoque, por una parte, en el diseño de hipertextos, software del cual aprender, y de programas para que los estudiantes representen conocimiento, software con el cual aprender, y, por otra, en la identificación de representaciones de conocimiento elaborados parlas estudiantes de pedagogía y de profesores en ejercicio.

Las preguntas que hacemos a un documento, cuando tenemos este acercamiento metodológico, es la misma que hacemos al profesor, al experto o al profesor novel. Por una parte, preguntamos por el esquema ontológico $y$, por otra, por el contenido del esquema. $Y$ cuando estamos en actividades de formación distinguimos das niveles: el 
nivel de conocimiento objeto o contenido de la representación y el metaconocimiento o conocimiento de la estructura de representación y su dominio.

Las consecuencias metodológicas del enfoque ontológico, entonces, se concretan en un enfoque de representación de conocimiento para el diseño de software, y en una aproximación metodológica al estudio de la formación de competencias cognitivas y metacognitivas.

\section{Sistemas Q}

Nuestro interés por el estudio de sistemas conceptuales complejos, nos ha acercado a un sistema basado en ordenamientos sucesivos de subconjuntos de proposiciones escritas en tarjetas y que forman parte de una pila más grande, la cual representa el sistema completo de conceptos. Las ordenaciones parciales y sucesivas, hechas con base en relaciones como, "estar más de acuerdo" o "la más adecuada", dan como resultado la ordenación de la pila total. Diez y seis proposiciones, por ejemplo, pueden ser ordenadas en dos pilas tomando parejas de tarjetas y colocando a la izquierda aquella con la que estoy más de acuerdo y la otra a la derecha; luego, cada una de ellas da lugar a una segunda partición para cuatro pilas de 4 tarjetas. Las tarjetas adquieren valor según el lugar que ocupan en la pila.

El cruce de variables de los sujetos con características del contenido de las proposiciones, permite hacer análisis estadísticos. Por ejemplo, nos preguntamos si hay diferencia en las puntuaciones sobre locus de control entre niños y niñas, o según el nivel educativo.

En otra forma de aplicación, se valoró la capacidad de liderazgo de los estudiantes al contrastar ordenamientos individuales con ordenamientos en parejas o tríos. Este acercamiento permite al grupo orientar el diseño de software para administrar este tipo de sistemas.

\section{Proyectos desarrollados y aportes teóricos}

Las tensiones entre heteronomía y autonomía se manifiestan en las organizaciones sociales y tienen repercusiones muy amplias en los diferentes retos de la vida humana. Las personalidades y las organizaciones mismas, cuando tienen tendencias dominantes hacia la heteronomía, acuden a fuerzas externas para resolver sus problemas, en contraste con las personalidades autónomas más involucradas en la construcción de soluciones.

Nuestro grupo decidió, desde sus inicios, comprometer sus esfuerzos en la comprensión de la autonomía y en la generación de modelos educativos para formarla.

El aprendizaje autónomo y significativa en procesos de razonamiento espacial, como línea ciue ha venido siguiendo el grupo investigador, da origen a los siguientes proyectos:

\section{a. Ambiente computa rizado para el aprendizaje autodirigido del diseño}

Cofinanciado por Universidad Pedagógica Nacional y Colciencias (Maldonado et al., 2000), estudia la relación entre autoevaluación y aprendizaje, haciéndose tres preguntas: si la precisión de la autoevaluación aumenta en función de la retro-información; si la mayor precisión en la autoevaluación favorece la retención de conceptos; y si existen 
diferencias en el aprendizaje entre estudiantes que auto-evalúan su aprendizaje y los que no lo hacen. Para tal efecto, desarrollamos un programa de computador cuyo dominio fueron los conceptos de movimiento, equilibrio y significado en el diseño gráfico. Este interés es consecuencia de nuestro compromiso con la educación en tecnología. Combina metodología de análisis de protocolos con el diseño experimental estadístico. Los resultados de este trabajo muestran que la retroinformación no incide en la precisión de la autoevaluación en el corto plazo, pero sí en una reducción del tiempo requerido para hallar la solución al problema y que la precisión de la autoevaluación correlaciona con la retención de conceptos en el mismo contexto. La autoevaluación, por otra parte, se manifiesta como un activador poderoso de estrategias de solución de problemas, en tanto los estudiantes más exitosos evalúan sus estrategias de búsqueda.

\section{b. Razonamiento espacial y aprendizaje del diseño}

Este proyecto, copatrocinado por la Universidad Pedagógica Nacional y el DEP (Maldonado et al. 1999), presenta el concepto de activador de juicio de metamemoria como una comunicación que induce a los estudiantes a valorar su nivel de aprendizaje y el contenido de su memoria de largo plazo. De igual forma, se evalúa el efecto de la sugerencia de estrategia en la solución de problemas. Desarrollamos 9 juegos de computador sobre temas de razonamiento espacial y diseño. En esta investigación, se combina un análisis cuantitativo con uno cualitativo. En el primero, se aplican dos métodos estadísticos, un análisis factorial de varianza para evaluar el efecto de los activadores de juicios de metamemoria y la sugerencia de estrategia sobre las variables eficacia, eficiencia y rendimiento en forma independiente y un análisis de regresión múltiple para evaluar la generalización de estrategias entre parejas de juegos. El análisis cualitativo se hace con base en la ejecución de simuladores. Se encuentra que, tanto los activadores de juicios de metamemoria como la sugerencia de estrategias, tienden a ser más efectivos después de alguna experiencia de aprendizaje, además, que la combinación de estos dos factores genera un efecto positivo de interacción sobre la eficacia y la eficiencia en la solución de problemas. La síntesis de resultados nos llevó a visualizar el surgimiento de micra-sistemas motivacionales autónomos, que se manifiestan como retos personales que toman fuerza progresiva con el proceso de resolver problemas. Por otra parte, constatamos que las personas tendemos a resolver problemas a partir de nuestros esquemas previos y que estamos dispuestos a recibir sugerencias de estrategias nuevas, sólo después de probar su ineficacia.

\section{c. Razonamiento espacial y aprendizaje significativo}

La financiación del proyecto estuvo a cargo de la Universidad Pedagógica Nacional y Colciencias (Maldonado et al., 2000). A este estudio llegamos con varias preguntas por resolver. Por una parte, pensábamos que las estrategias fuertes eran aquellas que se podían generalizar entre problemas y las que, por otra parte, mostrarían mayor variedad. También, teníamos la intuición de que la comprensión y profundidad en el razonamiento basado en sistemas conceptuales representados por una red semántica estructurada, podría estar relacionado con la habilidad para resolver problemas de descubrimiento en el dominio de conocimiento de la red. $Y$ finalmente, estábamos interesados en ver si los profesores que hacían análisis de procesos de solución de problemas adquirían la tendencia a monitorear estos procesos y no tanto en verbalizar el contenido de la asignatura en sus clases.

Diseñamos redes semánticas estructuradas y con base en ellas elaboramos hipertextos sobre temas de razonamiento espacial, arte y diseño. Luego, desarrollamos un software 
evaluador de la comprensión conceptual definida operacionalmente como conexiones de cada nodo con otros nados y la profundidad de las relaciones como la capacidad de manejar varios niveles de la estructura para hacer inferencias siguiendo la jerarquía de la red semántica.

Posteriormente, configuramos cuatro versiones de juegos de descubrimiento que combinan los activadores de juicios de metamemoria con sugerencias de estrategias en dos modalidades: tiempo y eventos. Este proceso maneja dos escenarios: uno, incluye sugerencia fija de estrategias y otro, sugerencia adaptativa. De esta manera, replicamos los hallazgos del estudio anterior.

Finalmente, se diseñaron agentes simuladores del proceso de solución que hacían seguimiento del proceso de solución ejecutado por cada sujeto.

En una primera parte, se trabajó con estudiantes de décimo y undécimo grado de bachillerato y se hallaron los resultados.

En una segunda parte, se trabajó con cuatro grupos de profesores, los cuales estuvieron en una de las siguientes condiciones: 1. Resolver juegos de descubrimiento y luego enseñarlas a sus alumnos en sus salones de clase. 2. Resolver los problemas y luego estudiar la simulación de la solución ejecutada, para luego ir a enseñar a sus alumnos. 3. Resolver los juegos, estudiar simulaciones de otro jugador y luego ir a enseñar. 4. Estudiar las simulaciones de otros y luego ir a enseñar.

Este estudio generó información valiosa sobre varios tópicos. Contrario a lo que nosotros pensábamos, las estrategias fuertes tienen variedad restringida. Lo característico es la homogeneidad. Los estudiantes muy hábiles usan pocas estrategias, pero son consistentes en su uso. La generalización opera de varias maneras: entre juegos similares, hay generalización entre estrategias usadas en niveles avanzados del juego anterior con respecto a las primeras etapas del siguiente juego; entre juegos diferentes, hay generalización de estrategias de exploración en las primeras etapas de los pares de juegos. Cuando los juegos son diferentes, las estrategias fuertes consolidadas en las últimas etapas del juego anterior disminuyen la eficacia del siguiente juego en sus primeras etapas.

Por otra parte, se encontró que los estudiantes aprenden más fácilmente las categorías generales que los contenidos particulares y relacionar conceptos es la tarea más difícil. Por otra parte, el conocimiento estructural o de relaciones es un buen predictor en la solución de problemas de descubrimiento, seguido por el aprendizaje de categorías.

La combinación de sugerencias adaptativas, cuando el sujeto las necesita, con activadores de juicios de metamemoria, inciden significativamente en el rendimiento. Las sugerencias adaptativas basadas en tiempo, influyen de manera más temprana en la eficiencia en la solución de problemas. Por otra parte, la sugerencia adaptativa combinada con activadores de juicios de metamemoria, basados en eventos, incide en mayor eficacia y variedad de estrategias usadas en etapas avanzadas.

Se analizan como componentes críticos en la formación de la capacidad de monitorear procesos de estudiantes, por parte de los profesores, los siguientes: la experiencia con problemas similares a los que enseña, el nivel de competencia como solucionador de los problemas enseñados, el nivel metacognitivo o conocimiento de sus propios procesos y el nivel de conocimiento por observación de los procesas de solución seguidos por otros. 
d. Construyendo la autonomía en el aprendizaje de las áreas de ciencias de la naturaleza, la matemática, el español y la tecnología, en sexto grado de educación básica

Patrocinados por la Universidad Pedagógica Nacional y el IDEP generamos un proyecto de innovación que tuvo como objetivo crear un modelo que incorporara los hallazgos de los estudios experimentales y enfrentara el reto de formar para la autonomía y la colaboración en aulas escolares (Ortega et al., 2001).

El modelo integró los siguientes elementos: un ambiente de tarea compuesto por problemas centrales y fuentes de información, especialmente en formato de texto; trabajo individual, donde el estudiante identificaba el problema, formulaba sus juicios de metamemoria y hacía sus búsquedas de solución; un ambiente de trabajo colaborativo de contrastación y ajuste de solución; un ambiente para la autoevaluación y, finalmente, un ambiente de acreditación de logros de aprendizaje.

En el desarrollo de la experiencia participaron dos cursos de grado sexto de educación básica, uno de niños en jornada de la mañana y otro de adultos en jornada nocturna, en las áreas de matemática, español, ciencias y tecnología.

El proyecto puso a prueba, también una estrategia para hacer transferencia de resultados de investigación al aula, involucrando plenamente a los profesores de los colegios en el proceso. El modelo, en este sentido, se basó en las siguientes etapas: 1. los investigadores elaboran un modelo; 2. los investigadores socializan el modelo con los profesores regulares y hacen los ajustes pertinentes; 3. el profesor es observador del proceso y analiza la información registrada con los investigadores, en tanto otra persona dirige las sesiones de aula; 4. el profesor asume la dirección del proceso.

Los estudiantes, inicialmente, muestran alta dependencia de las orientaciones del profesor. Frecuentemente, tienen dificultades tanto en el trabajo individual como colaborativo. El aprendizaje de contenidos fue también lento en sus inicios. Después del primer mes de trabajo, se hizo evidente la consolidación de la capacidad para definir metas, cumplir agendas y hacer búsquedas de información. A partir del tercer mes, los estudiantes avanzaron más rápido en el aprendizaje de contenidos que los otros cursos del mismo colegio e invirtieron más tiempo en el estudio de las asignaturas. Al terminar el primer semestre yen el siguiente, obtuvieron un rendimiento superior en todas las asignaturas en comparación con los demás cursos del mismo grado.

Los profesores, por otra parte, mantuvieron la innovación en la segunda parte del año y la enriquecieron con materiales, problemas y actividades pedagógicas.

\section{e. Relación simuladores y aprendizaje por colaboración en problemas de descubrimiento}

Con patrocinio de la Universidad Pedagógica Nacional y el DEP (Maldonado et al, 2001) se desarrolló un proyecto que tuvo como propósito estudiar el efecto de estudiar la simulación de los procesos de solución de problemas de descubrimiento, contrastando la condición de estudio individual con la colaborativa.

Se usó el software de simulación habilitado para hacer clasificación y optimización de procesos. Una vez terminado un juego, el simulador puede replicar el proceso a voluntad, tanto en su totalidad como por segmentos. También puede usar estrategias para reducir el número de eventos y el tiempo requerido para resolver el problema. 
Las conclusiones muestran que el estudio del simulador tiene efectos significativos en el desempeño del aprendiz y especialmente en la eficacia, la cual se relaciona con la calidad de las estrategias aplicadas. La curva de aprendizaje evoluciona más rápido que cuando no se usa el simulador. Por otra parte, la condición colaborativa tiene efectos motivacionales positivos, pero su efecto es menor que el efecto principal del uso del simulador.

\section{f. Ontologías y aprendizaje: software para representar y software para comprender}

Este proyecto, que se llevó a cabo con el apoyo financiero de la Universidad Pedagógica Nacional y Colciencias, (Maldonado, Ortega, Sanabria y Macías, 2002), tuvo como propósito comparar una estrategia constructivista basada en software con otra centrada en procesos de comprensión activada por un generador de preguntas y probar el efecto estructurante de la utilización de ontologías en la formación de competencias de noveles profesores de geografía.

En la representación, se consideran conjuntos de entidades que se proyectan en una concepción sobre redes de conceptos. Este nuevo enfoque potencia la representación del mundo como construcción de conocimiento y el desarrollo de dimensiones cognitivas del estudiante. El conocimiento de estructuras y su utilización en actividades de aprendizaje, constituye un nivel superior al del manejo de contenidos. Estas categorías constituyen un metaconocimiento que potencia el monitoreo de la formación de sistemas conceptuales y modelos mentales del mundo. La ontología se usó como un sistema de categorías orientadoras para identificar la representación que un agente hace del conocimiento cuando actúa como orientador de procesos de aprendizaje.

La investigación tuvo dos partes: en la primera, estudiantes de noveno grado estudiaron el contenido de tres unidades de aprendizaje sobre geografía de Colombia y luego fueron evaluados con el mismo instrumento. En la segunda, estudiantes de licenciatura en educación con especialidad en ciencias sociales, estudiaron estas mismas unidades y luego dirigieron clases en la modalidad de microclases.

La experimentación tuvo dos condiciones: en la primera, diseñamos un hipertexto y un agente de software generador de preguntas, al cual denominamos "Preguntón"; en la otra diseñamos materiales impresos con el mismo contenido textual que el hipertexto y un agente de software que usaba el estudiante para elaborar un hipertexto estructurado con base en estructura de marcos, al cual llamamos "Simas".

Las conclusiones del estudio muestran cómo un agente generador de preguntas lleva al estudiante a comprometer una actividad muy intensa en el proceso de lectura, y cómo la actividad constructiva es más estructurante que la sola lectura.

Por otra parte los estudiantes de licenciatura que estudiaron con base en el programa "Simas" desarrollaron clases más estructuradas y con un menor número de categorías ontológicas que sus contrapartes, que estudiaron apoyándose en hipertextos y en un agente generador de preguntas. Las diferencias se acentuaron en las últimas sesiones.

\section{g. Desarrollo de competencias en las áreas de tecnología y matemáticas a través de marcos conceptuales}

Con base en los estudios experimentales anteriores, especialmente del proyecto sobre ontologías y aprendizaje, y orientados por el trabajo sobre construcción de autonomía, 
desarrollamos un segundo proyecto en aula durante el año 2001 en el Centro Educativo Distrital Venecia, en la ciudad de Bogotá. Participó un curso de estudiantes del grado sexto, en las áreas de tecnología y matemáticas. Nos encaminamos a desarrollar y fortalecer las habilidades cognitivas, metacognitivas, colaborativas y motrices, bajo la estrategia de representación de conocimiento a través del Sistema de Marcos, apoyado por el software "Simas".

Los estudiantes centran su actividad en la representación de conocimiento a partir de las fuentes de información suministradas por el profesor y otras seleccionadas parlas estudiantes con orientación de su profesor. El resultado se concreta en un hipertexto que muestra el estado final del proceso de aprendizaje. En la dinámica del desarrollo se conservan los parámetros de la primera innovación.

En la siguiente gráfica se muestra la estructura de una unidad de aprendizaje y se identifican cada una de las partes que la componen.

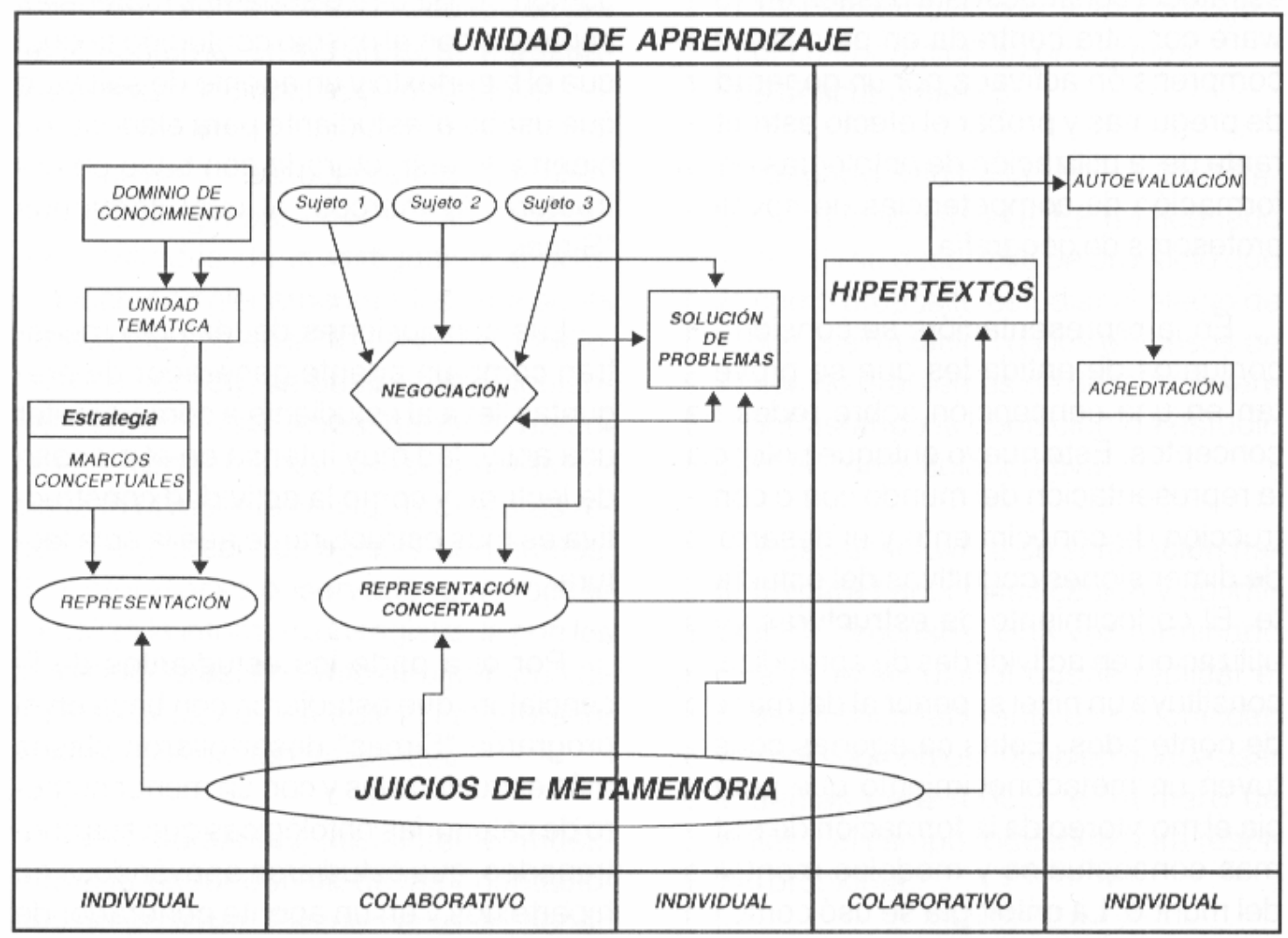

Los resultados de esta innovación se pueden resumir en los siguientes aspectos:

Los estudiantes, a medida que avanzaban en el proceso, desarrollaban la competencia de representación de conocimiento, lo cual les permitió procesar información en forma más eficiente. Esta competencia se extendió a otras asignaturas como capacidad de estructurar respuestas a problemas en diferentes situaciones.

Los estudiantes desarrollaron habilidades para buscar información en la biblioteca, en los laboratorios y en enciclopedias multimediales, entre otros. 
Los estudiantes desarrollaron la capacidad de planear su trabajo y desarrollarlo autónomamente. Los estudiantes desarrollaron alta motivación al trabajo académico, que les llevaba a invertir espontáneamente mucho tiempo en su desarrollo.

La habilidad de subdividir un problema en subproblemas, se constituyó en una estrategia fuerte en la solución. Los estudiantes adquirieron elementos de juicio para estructurar de una forma holística la información y con el nivel de detalle y especificidad requerido en cada problema.

La representación de conocimiento mediante el sistema de marcos, se convierte en el escenario propicio para desarrollar las habilidades de adquisición, organización y selección de la información, identificación de relaciones causa efecto, descomposición de un sistema en subproblemas.

\section{Aportes tecnológicos}

Los aportes del grupo, a nivel tecnológico, se concretan en el desarrollo de los prototipos de programas de computador utilizados en el desarrollo de los diferentes experimentos referidos en este documento. Algunas de estas piezas tienen la concepción de programas de los cuales se aprende como es el caso de los hipertextos pero, la mayoría tienen el enfoque de programas con los cuales se aprende. El siguiente es el resumen de estos aportes.

1. Juegos de descubrimiento: son piezas que presentan un problema para ser resuelto. Tienen muy pocas instrucciones y requieren observación y razonamiento de parte del usuario. Típicamente se programan a partir de la identificación de un estado de entrada, un operador, operandos y transiciones de estado. La solución del problema es un estado meta especificado en la formulación del problema.

2. Hipertextos con estructura de marcos. Estos hipertextos estructurados han tomado como temáticas la historia de la grúa, la historia de los engranajes, la relación entre arte y diseño y la geografía colombiana.

3. Simuladores de procesos. Hacen seguimiento paso a paso del proceso seguido por un usuario en la solución de un problema, permiten replicarlo y optimizarlo.

4. "Simas": es una estructura que sirve de base para que el usuario cree un hipertexto, siguiendo una estructura de marcos.

5. Generadores de preguntas: toman como fuente un hipertexto estructurado, formulan una pregunta y desarrollan un breve diálogo con el usuario.

6. Agentes de software para el juego de roles: tomando como referencia un juzgado, simulan papeles como el de juez, denunciante o sindicado.

7. Sistemas $\mathrm{Q}$ : son programas que toman como fuentes hipertextos y generan baterías de proposiciones y piden a un usuario que las ordenen según un criterio. 


\section{Conclusión: prospectiva}

Las búsquedas del grupo conservan el interés por la formación de la autonomía, por su gran potencial en la era de la información y por la riqueza teórica y social. De las nuevas generaciones de profesores se esperan competencias para trabajar en los escenarios que proveen las nuevas tecnologías de la información, enriquecerlas y comprender a profundidad la dinámica educativa que se genera, tanto en términos de aprendizaje como de construcción cultural.

En la actualidad, los esfuerzos están centrados en los siguientes proyectos:

1. Juegos de roles en el aprendizaje de los derechos fundamentales de la Constitución Colombiana.

2. Sistemas $Q$ y construcción de sistemas conceptuales complejos.

3. Generadores de preguntas en la comprensión de hipertextos.

4. Validación de un modelo de formación de competencias docentes para la enseñanza usando entornas virtuales de aprendizaje.

\section{Bibliografía}

Davidson, J. E. "Deuser, R. \& Sternberg, R. J. (1994) The role of metacognition in problem solving", in: Metcalfe, Jane and Shimamura, Arthur P. (Eds.) Metacognition. Cambridge, MA: The MIT Press. pp. 207-226.

Ericson, K. A. \& Clutcher, R. J. (1991) Introspection and verbal reports on cognitive processes - twa studies of thought processes: a response to howe", en: New Ideas in Psychology, 9, pp. 57-71.

Ericson, K. A. \& Simon, H. A. (1993) Protocol analysis: verbal reports as data. Cambridge, MA: The MIT Pres. Second Edition.

Maldonado G., L. F. (1989) The effect on performance and learner-sequencing decisions of instructional curriculum maps in a hypertext environment. Doctoral Dissertation, Florida State University. Dissertation Abstracts International.

Maldonado G., L. F. (1994) Análisis de protocolos: posibilidad metodológica para el estudio de procesos cognitivos en personas con discapacídad. Bogotá, Universidad Pedagógica Nacional, Curso Internacional sobre Tecnologías de la Rehabilitación para Personas con Discapacidad.

Maldonado G., L. F. y Ochoa, M. L. (1984) Proyecto de investigación para el desarrollo de modelos de diseño y evaluación de materiales didácticos a partir de las teorías de la cibernética, la informática y la comunicación. Bogotá, Universidad Pedagógica Nacional. 
Maldonado G., L. F. y Andrade L. (1996a) Learner-Controlled, computer-based environment for developing design capabilities. second jerusalem international science \& tecnology education Conferonce JISTIC 96. Enero 8-11 de 1996. Proceedings.

Maldonado G., L. F. y Andrade L. (1996b) Ambiente computarizado para el aprendizaje autodirigido del diseño - ACA2-. Bogotá, Memorias del Primer Congreso Internacional de Educación y Tecnología EDENTEC 96.

Maldonado G., L. F., Carrillo G, I., Monroy, L. B. y Terrero G., A. (1977) Diseño y comparación de cuatro métodos de enseñanza en un curso introductorio de psicología del aprendizaje. Bogotá, Universidad Pedagógica Nacional.

Maldonado G., L. F., Ortega, N., Fonseca, O. H., Sarmiento, L. C., González, D. N., Macias, M. D. e Ibañez, I. J. (2001 C) La relación Simuladores y colaboración en el aprendizaje. Bogota, Instituto para la Investigación Educativa y el Desarrollo Pedagógico y Universidad Pedagógica Nacional.

Maldonado G., L. F., Fonseca, R., O.H. Ibáñez, I., J. Macías, Ortega, N., Rubio, Sanabria. R., LB. (1999) Metacognición y razonamiento espacial en juegos de computador. Bogotá, Instituto para la Investigación Educativa y el Desarrollo Pedagógico y Universidad Pedagógica Nacional.

Maldonado G., L. F. y Andrade, L. (2001a) Ambiente Computa rizado para el aprendizaje autodirigido del diseño ACA2. Bogotá, Universidad Pedagógica Nacional y Colciencias.

Maldonado G., L. F., Ortega, N., Fonseca, R., OH., Rubio, 8., M. R., Ibáñez, I., J. y Macías. M., D. (2001 B). Razonamiento espacial y aprendizaje significativo: profesores y alumnos frente a los juegos de descubrimiento basados en com putador. Bogotá, Universidad Pedagógica Nacional y Colciencias.

Maldonado O., L. F., Ortega, N., Sanabria, LB., Macías. M., D. (2002) Ontologías y aprendizaje: software para representar y software para aprender. Bogotá, Universidad Pedagógica Nacional y Colciencias.

Metcalfe, J. and Shimamura, A. P. (Eds., 1994) Metacognition. Cambridge, MA: The MIT Press. Preface.

Minsky, M. (1974). A framework for representing knowledge, in Haugeland, J. (1984), editor, Mmd Design, pagos 95-128, MIT Press, Cambridge MA. Reprinted in Brachman and Levesque (Eds).

Nelson T. O. \& Narens, L. (1990) "Metamemory: a theoretical framework and new findings, In G. Bower (De.). The Psychology of Learning and Motiva tion (Vol. 26). New York, Academic Press.

Newell, A. \& Simon, H. A. (1972) Human problem solving. englewood cliffs, NJ: Prentice Hall.

Ortega, N. (1997) Razonamiento espacial y solución de problemas en maestros de ciencias sociales y matemáticas. Bogotá, D.C., CINDE - UPN, Tesis para obtener el Título de Magíster en Educación. 
Sowa, J. F. (1986) Conceptual structures: information processing in mmd and machine. Reading MA: Addison Wesley Publishing Company.

Sowa, J. F. (1987) "Semantics Networks", In Encyclopedia of Artificial Intelligence. New York, N.Y., Edit. John Willoy and Sons. Edited by Stuart C. Shapiro. 